'Sección Gastroenterología,

Departamento de Medicina

Interna, Hospital Clínico

Universidad de Chile. Santiago,

Chile

${ }^{2}$ Clínica Dávila, Santiago, Chile.

Apoyo financiero: ninguno.

Recibido el 17 de abril de 2016

aceptado el 23 de noviembre de 2016

Correspondencia a:

Dr. Zoltán Berger F.

Hospital Clínico Universidad de

Chile.

Departamento de Medicina,

Sección Gastroenterología

Santos Dumont 999,

Independencia, Santiago, Chile.

Código Postal: 8380456

Teléfono: 56-2-229788350,

Fax: 56-2-229788348

berger.zoltan@gmail.com

\section{¿Es la pancreatitis crónica una enfermedad rara en Chile? ¿Subdiagnóstico, baja prevalencia o ambos?}

\author{
ZOLTÁN BERGER F. ${ }^{1,2}$, CARLA MANCILLA A. ${ }^{1}$
}

\section{Chronic pancreatitis. Retrospective review of 121 cases}

Background: Chronic pancreatitis $(C P)$ is a rare disease in Chile, without a clear explanation for this low prevalence. Aim: To analyze the characteristics of our patients with pancreatitis. Material and Methods: Retrospective analysis of a database of patients with pancreatitis of a clinical hospital. Morphological proof of diagnosis (calcifications/calculi, alterations of ducts, local complication or histology) was obtained for every patient. History of acute pancreatitis was recorded and exocrine-endocrine function was assessed. Results: We retrieved information of 121 patients with pancreatitis ( 86 males) in a period of 20 years. The number of cases increased markedly every five years. The calculated incidence and prevalence was 0.8/100,000/year and 6/100,000, respectively. Pancreatic calcifications were initially observed in 93 patients and became evident during the follow-up in another six patients. Severe pain or local complications occurred in 27 patients, requiring surgery in 10 or endoscopic treatment in 15 . During the years of follow-up, 55 patients were free of symptoms. Exocrine and endocrine insufficiency was demonstrated and treated in 81 and 67 patients, respectively. Alcoholic etiology was evident in $40 \%$ of patients. In $29 \%$ no etiology was identified. Mapuche origin was exceptional. Conclusions: Late diagnosis of CP is common, since most of our patients presented with advanced stages. Even though $C P$ is increasingly diagnosed in our hospitals, the number of cases is still far fewer when compared to other countries. Underdiagnosis alone cannot explain this difference and genetic factors might be of importance.

(Rev Med Chile 2016; 144: 1544-1552)

Key words: Delayed Diagnosis; Epidemiologic Factors; Genetic Predisposition to Disease; Pancreatitis, Alcoholic; Pancreatitis, Chronic.
L a pancreatitis crónica (PC) es considerada una enfermedad rara, sino excepcional, en paises latino-americanos, con excepde enfermedades pancreáticas de $1979^{4}$ se comunicaron pocos casos desde Argentina y Chile, predominando las formas no calcificadas. En el año 2000 se organizó en Chile un encuentro latinoamericano de enfermedades pancreáticas. En esa ocasión solamente los representantes de México y Brasil refirieron tener experiencia significativa en PC. En Chile se han publicado pocas series de casos, con bajo número de pacientes, incluso en una de las más recientes ${ }^{5}$.

La primera interrogante es el diagnóstico: ¿Es posible que esta diferencia se deba solamente a falencias en el diagnóstico? Las deficiencias en los sistemas de salud en América Latina son conoci- 
das. Sin embargo, el acceso a los procedimientos imagenológicos modernos ha aumentado en las últimas décadas, permitiendo el diagnóstico de la enfermedad avanzada con cambios ductales y calcificaciones. Por el contrario, el diagnóstico precoz es difícil con imágenes y los tests de medición directa de la función exocrina, de mayor sensibilidad, prácticamente no se encuentran disponibles.

¿Podrían diferencias nutricionales o en el consumo de tabaco y alcohol explicar la menor prevalencia, especialmente considerando que la pancreatitis aguda biliar y la enfermedad hepática alcohólica son prevalentes en Chile y más frecuentes que en otros países?6.

En este artículo presentamos nuestra experiencia de 20 años, donde analizamos las características de la PC. A nuestro entender corresponde al seguimiento clínico de mayor número de pacientes en Chile; algunos aspectos de esta observación nos permiten plantear posibles diferencias con el resto del mundo.

\section{Pacientes y Método}

Análisis retrospectivo de historia clínica de pacientes incluidos en una base de datos registrados en forma prospectiva y permanente. El análisis fue realizado entre diciembre de 2015 y febrero de 2016. Los pacientes fueron inicialmente evaluados en forma ambulatoria u hospitalizada con diagnóstico establecido o sospecha de enfermedad pancreática y controlados posteriormente. Se obtuvo confirmación morfológica de la pancreatitis crónica (PC) por TAC (tomografía axial computada) en todos los pacientes, resonancia magnética (RM) en 96 de ellos. Consideramos confirmado el diagnóstico morfológico de PC al detectar atrofia del parénquima con dilataciones típicas del conducto pancreático principal ("cadena de lagos") y de ramas secundarias, con o sin calcificaciones parenquimatosas o cálculos pancreáticos intraductales. Endosonografía (EUS) fue realizada en sólo 11 pacientes, en 9 de ellos para definir necesidad de cirugía o intervención endoscópica. De los 10 pacientes operados, se realizó resección pancreática en 8, los hallazgos histológicos confirmaron el diagnóstico de PC, con extensa fibrosis, atrofia de células acinares e infiltración inflamatoria leve. Se registró la historia de pancreatitis aguda. La función exocrina se evaluó con la determinación de elastasa fecal, que se realizó mediante técnica de Elisa (Schebo Biotech, Giessen, Alemania) ${ }^{7}$. El valor normal de la elastasa fecal es sobre $200 \mu \mathrm{g} / \mathrm{g}$ de deposición, niveles entre 100 y $200 \mu \mathrm{g} / \mathrm{g}$ representan insuficiencia exocrina moderada y menores de $100 \mu \mathrm{g} / \mathrm{g}$ demuestran insuficiencia severa. El diagnóstico de diabetes mellitus se estableció con los criterios conocidos ${ }^{8}$ : encontrando glicemia en ayunas $>126 \mathrm{mg} / \mathrm{dl}$ al menos dos ocasiones. No se realizó búsqueda de intolerancia a glucosa con carga oral de glucosa. Los pacientes fueron interrogados por dos diferentes médicos en dos diferentes ocasiones para evaluar su consumo de alcohol. Durante la evolución los pacientes fueron hospitalizados y recibieron manejo endoscópico o quirúrgico según necesidad. La historia médica fue analizada de forma retrospectiva luego de la aprobación por el Comité de Ética del Hospital Clínico Universidad de Chile y el de Clínica Dávila.

\section{Resultados}

En este período de 20 años, reunimos 121 pacientes con PC, 86 hombres $(\mathrm{H})$ y 35 mujeres $(\mathrm{M})$. De estos, 34 (25 H-9 M) eran menores de 35 años al momento del diagnóstico y 10 de ellos (5H-5M), menores de 25 años (Tabla 1). El número de casos diagnosticado por períodos de 5 años aumentó marcadamente (Figura 1). Noventa y tres pacientes $(77 \%)$ presentaban calcificaciones pancreáticas al momento del diagnóstico y estas aparecieron en 6 pacientes más (5\%) durante el seguimiento. Hasta la fecha solamente 22 de nuestros pacientes (18\%) no presentan calcificaciones.

Del grupo estudiado, 48 pacientes pertenecen a las comunas del área norte de Santiago que se atienden en nuestro hospital, donde también se registró un aumento en el número de casos (Figura 2). Según el censo 2014, el área norte de Santiago (incluyendo las comunas Conchalí, Huechuraba, Independencia, Recoleta y Quilicura) cuenta con 666.195 habitantes. Con estos datos calculamos una incidencia de 0,8 casos por 100.000 habitantes/año para el período $2010-2015$ y una prevalencia de 6,1 casos por 100.000 habitante para el período 1996-2014.

La etiología fue el consumo de alcohol en 40\% de nuestros pacientes ( $43 \mathrm{H}-6 \mathrm{M})$, quienes relataron un consumo de más de 5 bebidas alcohólicas al día. Otros 17 pacientes (14\%) consumían 
Tabla 1. Características epidemiológicas

\begin{tabular}{|c|c|c|c|}
\hline & $\begin{array}{l}\text { Hombre } \\
(n=86)\end{array}$ & $\begin{array}{c}\text { Mujer } \\
(n=35)\end{array}$ & $\begin{array}{c}\text { Total } \\
(n=121)\end{array}$ \\
\hline \multicolumn{4}{|l|}{ Edad (años) } \\
\hline$<35$ & $25(29 \%)$ & $9(26 \%)$ & $34(28 \%)$ \\
\hline$>35$ & $61(71 \%)$ & $26(74 \%)$ & 87 (72\%) \\
\hline \multicolumn{4}{|l|}{ Etiología } \\
\hline Alcohol & $43(50 \%)$ & $6(17 \%)$ & $49(40 \%)$ \\
\hline Posible & $13(15 \%)$ & $4(11 \%)$ & $17(14 \%)$ \\
\hline Idiopática & $22(26 \%)$ & $13(38 \%)$ & 35 (29\%) \\
\hline Probable genética & $1 \quad(1 \%)$ & $2(6 \%)$ & $3(2,5 \%)$ \\
\hline Autoinmune & $2 \quad(2,3 \%)$ & $1 \quad(3 \%)$ & $3(2,5 \%)$ \\
\hline Recurrente & $4 \quad(4,7 \%)$ & $2 \quad(6 \%)$ & $6 \quad(5 \%)$ \\
\hline Obstructiva & $1 \quad(1 \%)$ & $5(14 \%)$ & $6 \quad(5 \%)$ \\
\hline Otra (hipercalcemia) & - & $2 \quad(6 \%)$ & $2(2 \%)$ \\
\hline \multicolumn{4}{|l|}{ Tabaquismo } \\
\hline Activo & $28(33 \%)$ & $9(26 \%)$ & 37 (30\%) \\
\hline Suspendido & $31(36 \%)$ & $11(31 \%)$ & $42(35 \%)$ \\
\hline Nunca & $27(31 \%)$ & $15(43 \%)$ & $42(35 \%)$ \\
\hline
\end{tabular}

Los pacientes fueron entrevistados para obtener datos de historia médica y de sus hábitos. La PC fue considerada idiopática si no pudimos detectar ninguna causa conocida en los antecedentes.

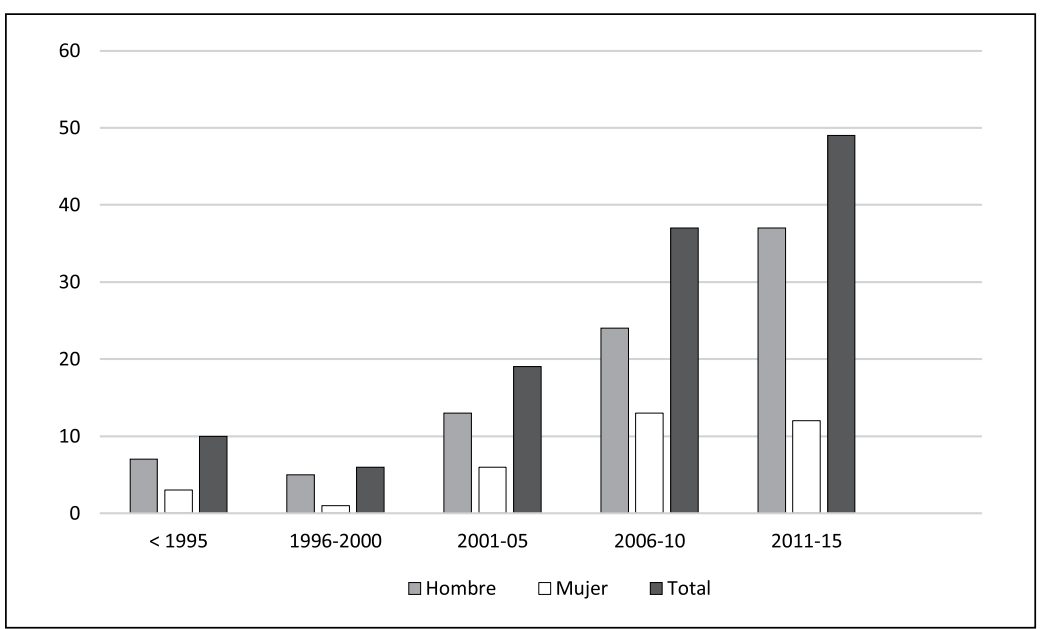

Figura 1. Diagnóstico de pancreatitis crónica en períodos de 5 años. alcohol regularmente, pero en cantidad considerablemente menor. Al diagnóstico, 37 pacientes eran fumadores activos (30\%), 42 (35\%) habían fumado antes y $42(35 \%)$ nunca habían fumado. Si bien no se estudiaron mutaciones, es probable que intervinieran factores genéticos en 4 casos (3,3\%): padre e hija, tía y sobrina. Factores autoinmunes se demostraron en 3 casos (2,5\%). En 29\% de los pacientes $(22 \mathrm{H}-13 \mathrm{M})$ no se pudo plantear etiología (Tabla 1). Respecto a la ascendencia, solamente 2 pacientes tuvieron origen mapuche por línea materna y ninguno por ambas líneas.

Los pacientes más jóvenes requirieron más hospitalizaciones para manejo del dolor y tera- 


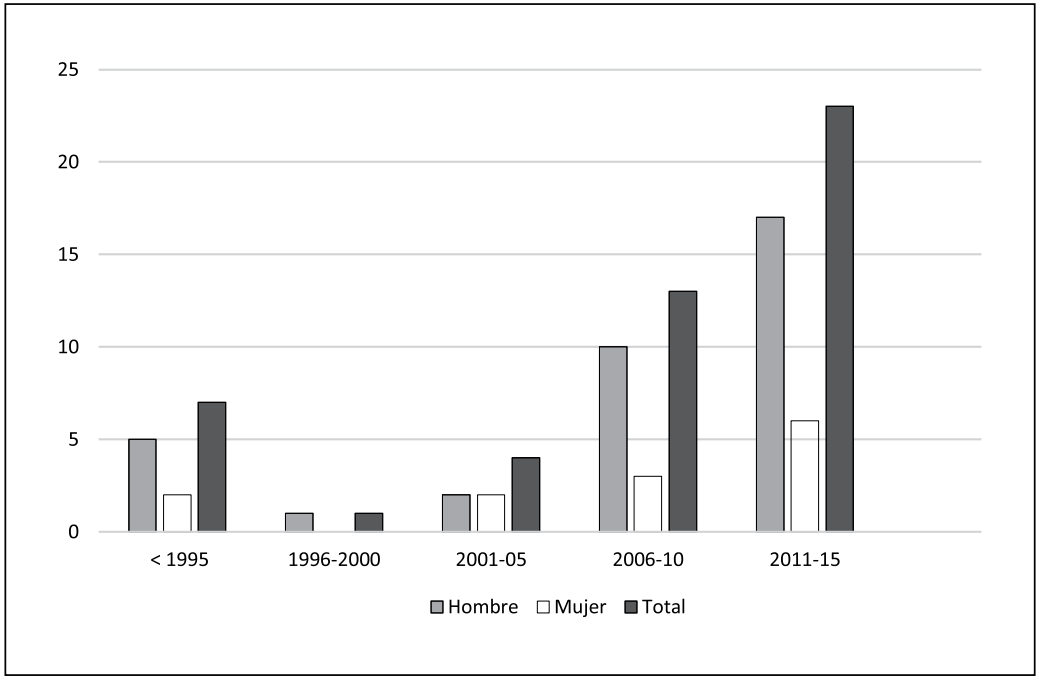

Figura 2. Diagnóstico de pancreatitis crónica en los barrios de Norte de Santiago en períodos de 5 años.

Tabla 2. Características clínicas y evolución

\begin{tabular}{|c|c|c|c|}
\hline & $\begin{array}{l}\text { Hombre } \\
(n=86)\end{array}$ & $\begin{array}{c}\text { Mujer } \\
(n=35)\end{array}$ & $\begin{array}{c}\text { Total } \\
(n=121)\end{array}$ \\
\hline \multicolumn{4}{|l|}{ Calcificación } \\
\hline Inicial & $66(77 \%)$ & $27(77 \%)$ & $93(77 \%)$ \\
\hline Seguimiento & $4(4 \%)$ & $2(6 \%)$ & $6(5 \%)$ \\
\hline No & $16(19 \%)$ & $6(17 \%)$ & $22(18 \%)$ \\
\hline \multicolumn{4}{|l|}{ Función exocrina } \\
\hline Esteatorrea clínica y/o elastasa fecal $<50$ ug/g & $44(51 \%)$ & $10(29 \%)$ & $54(45 \%)$ \\
\hline Elastasa $50-200 \mathrm{ug} / \mathrm{g}$ & $14(16 \%)$ & $13(37 \%)$ & $27(22 \%)$ \\
\hline Elastasa $>200 \mathrm{ug} / \mathrm{g}$ & $28(33 \%)$ & $12(34 \%)$ & $40(33 \%)$ \\
\hline \multicolumn{4}{|l|}{ Función endocrina } \\
\hline Diabetes insulino-requiriente & $21(24 \%)$ & $10(29 \%)$ & $31(26 \%)$ \\
\hline Diabetes sin insulina & $25(29 \%)$ & $11(31 \%)$ & $36(30 \%)$ \\
\hline Normal & $40(47 \%)$ & $14(40 \%)$ & $54(44 \%)$ \\
\hline Complicaciones locales & $11(13 \%)$ & $6(17 \%)$ & $17(14 \%)$ \\
\hline \multicolumn{4}{|l|}{ Dolor } \\
\hline Severo, invalidante & $7 \quad(8 \%)$ & $4(11 \%)$ & $11(9 \%)$ \\
\hline Moderado, ocasional & $38(44 \%)$ & 17 (49\%) & $55(45,5 \%)$ \\
\hline Sin dolor & $41(48 \%)$ & $14(40 \%)$ & $55(45,5 \%)$ \\
\hline Tratamiento endoscópico & $12(14 \%)$ & $3(9 \%)$ & $15(12 \%)$ \\
\hline Bloqueo plexo celíaco & $1 \quad(1,2 \%)$ & $1 \quad(3 \%)$ & $2(1,7 \%)$ \\
\hline Cirugía & $5 \quad(6 \%)$ & $5(14 \%)$ & $10(8 \%)$ \\
\hline Muerte & $9(10 \%)$ & $3(9 \%)$ & $12(10 \%)$ \\
\hline
\end{tabular}

La función exocrina fue medida por determinación de elastasa fecal. Glicemia y hemoglobina glicosilada fueron determinadas y el diagnóstico de diabetes fue establecido por los criterios conocidos. Certificados de defunción fueron obtenidos en Registro Civil. 

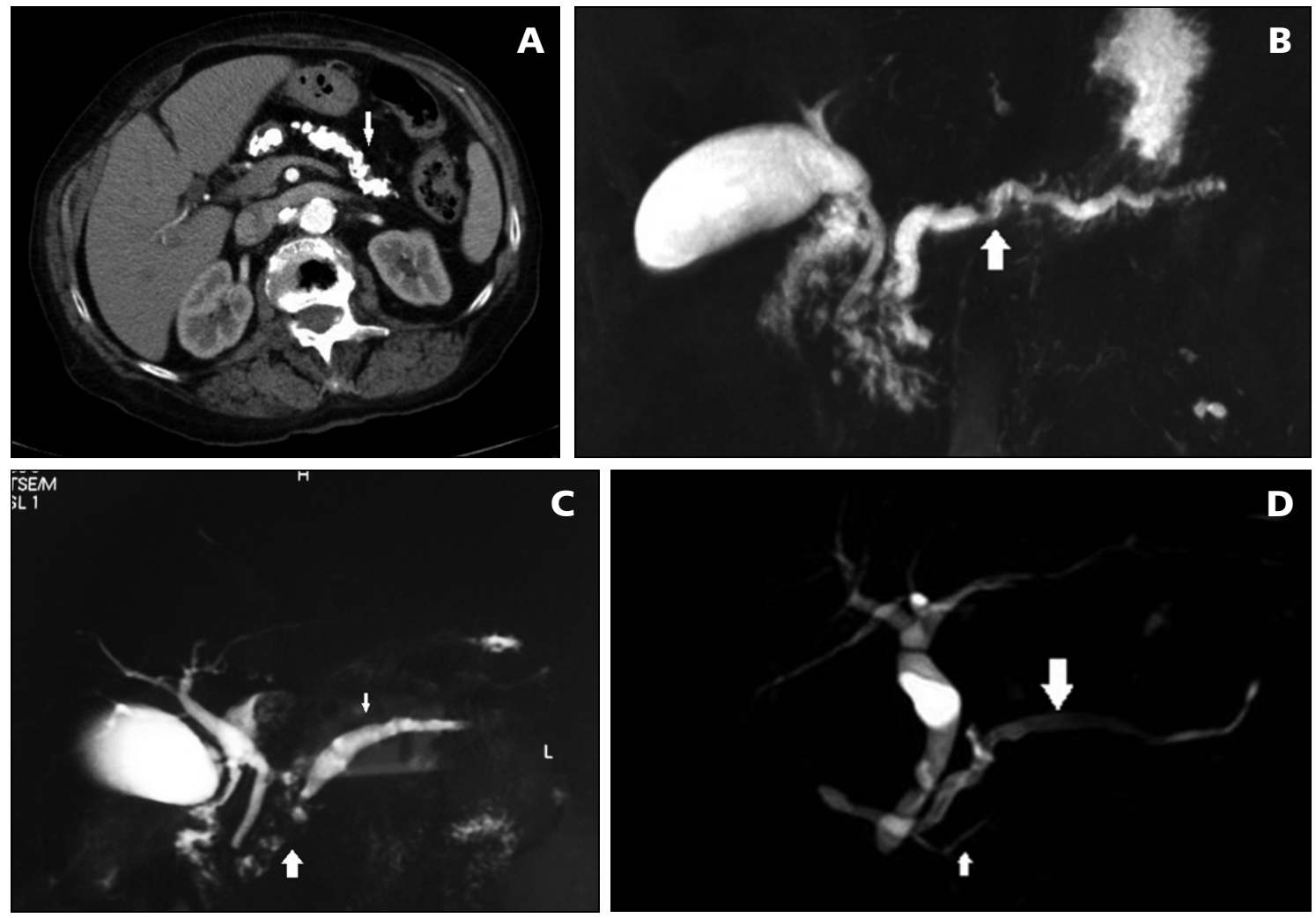

Figura 3. A. Mujer 73 años, mínimo dolor abdominal. Conducto pancreático ocupado por calcificación, cálculos (flecha), con atrofia casi completa del parénquima. B. Hombre 45 años, dolor abdominal moderado, insuficiencia exo-y endocrina. Resonancia magnética muestra dilatación típica del conducto pancreático, con contornos irregulares (imagen de "cadena de lagos"). C. Hombre 40 años. Colangioresonancia muestra que el conducto pancreático principal en la cabeza está ocupado por cálculos, que impiden visualización de este segmento (flecha) por ausencia de líquido (secreción pancreática). Dilatación de Wirsung en cuerpo y cola. D. Mujer de 50 años con dolor abdominal moderado. Resonancia magnética muestra páncreas divisum. Pancreatitis obstructiva, dilatación marcada del conducto dorsal ("Santorini"), que cruza el colédoco y desemboca por la papila menor (flecha ancha). El conducto ventral (flecha fina) es fino en la cabeza de páncreas y desemboca en la papila de Vater juntos con el colédoco.

pias invasivas, mientras los pacientes mayores se controlaron principalmente en forma ambulatoria. La insuficiencia exocrina $(67 \%)$ y endocrina $(55 \%)$ fueron frecuentes, incluso se observaron complicaciones crónicas de la diabetes en varios casos, con 3 casos de insuficiencia renal crónica, dos de los cuales fallecieron. La indicación de tratamiento invasivo fue por dolor intolerable, pancreatitis recurrente y complicaciones locales. Se realizó terapia endoscópica en 15 pacientes, cirugía en 10 y bloqueo del plexo celíaco en 2 . Por el contrario, 55 pacientes (45\%) presentaron una evolución indolora y sin eventos. En 15 pacientes de este grupo el diagnóstico se estableció en forma incidental como hallazgo de tomografía computa$\mathrm{da}$ (TC) indicada por otro motivo, luego de lo cual se confirmó la presencia de insuficiencia exocrina e incluso endocrina en algunos casos. Durante el seguimiento, 12 pacientes fallecieron $(10 \%)$, una de ellos, proveniente de un lugar lejano, certificada como cáncer de páncreas. Sin embargo, la paciente presentaba una TAC sin lesión focal 8 meses antes del fallecimiento y no encontramos datos en la historia que avalaran el diagnóstico de cáncer pancreático. Un paciente falleció de sepsis en relación a un pseudoquiste infectado, dos por insuficiencia renal crónica y 8 pacientes por causas no relacionadas a la enfermedad pancreática. 


\section{Discusión}

La PC es considerada una enfermedad rara por la gran mayoría de los gastroenterólogos y cirujanos digestivos en Chile. El objetivo de este artículo fue intentar responder las siguientes preguntas: ¿es realmente la PC una enfermedad rara o subdiagnosticada? ¿Hay alguna característica clínica específica en nuestros pacientes que dificulta el diagnóstico? ¿Hay factores en la dieta chilena, o en el consumo de tabaco/alcohol, o genéticos, que protegen al chileno de la PC?

El diagnóstico de PC ha aumentado en nuestro grupo durante los últimos años. La mayoría de nuestros pacientes se presentaron con formas avanzadas calcificantes, cuando el diagnóstico es fácil de establecer, con frecuencia por exámenes indicados sin sospecha de enfermedad pancreática. Considerando que según los escasos datos disponibles, la forma no calcificada fue predominante en Chile hace 40 años $^{4}$, podríamos hipotetizar que al menos el mismo número de pacientes se ha perdido por falta de diagnóstico. Sin embargo, es un problema más general, no solo válido en Chile, ya que el diagnóstico precoz de PC es complejo y deficiente en todo el mundo, como lo pone en evidencia el hecho de que un tercio de los casos no había sido diagnosticado en pacientes con PC encontrados en autopsias (3. Cit 3).

La sensibilidad y especificidad de los métodos diagnósticos es de gran importancia cuando hablamos de aspectos epidemiológicos de cualquier enfermedad. El diagnóstico de las formas avanzadas es fácilmente demostrable en TC o resonancia magnética (RM) por la presencia de calcificaciones, cálculos intraductales o dilatación irregular del Wirsung. Por el contrario, el diagnóstico de las formas leves, frecuentemente oligosintomáticas, es un desafío en todo el mundo, ya que no se dispone de un método ideal y de bajo costo. Las recomendaciones de la Asociación Americana de Páncreas fueron recientemente publicadas. Los tests de función pancreática no se utilizan para el diagnóstico precoz en la práctica clínica. La TAC tiene un buen valor predictivo positivo, pero no es lo suficientemente sensible. La RM tiene mejor sensibilidad, entregando información al mismo tiempo del parénquima comparable a la TAC y de los conductos, comparable a la colagio-pancreatografía retrógrada endoscópica (ERCP), sin los riesgos de un procedimiento invasivo. $\mathrm{La}$ adición de secretina endovenosa en la RM también da información semicuantitativa de la función exocrina, aumentando la sensibilidad del método y mejorando la visualización de los conductos menores, sin embargo, la secretina no está disponible en Chile. La endosonografía (EUS) es el examen más sensible pero menos específico para el diagnóstico de PC leve. Sin embargo, el sistema de salud chileno no financia este examen que es de alto costo y por esta razón no se recomienda para screening, ni en las guías recientes ${ }^{9}$. En nuestro medio, el diagnóstico morfológico se basa en la TAC y RM, reservando la EUS para casos en que se requiere terapia endoscópica o intervención quirúrgica. La ERCP tiene indicación exclusivamente terapéutica. Si la TAC o la RM no son concluyentes y la sospecha clínica persiste, nosotros repetimos la imagen en 1 año.

Como señalamos, la incidencia y prevalencia estimadas para el área norte de Santiago fueron de 0,8/100.000 habitantes/año y 6,1/100.000 habitantes, respectivamente. Yadav reportó 106 casos de PC en un período de 30 años en el condado de Olmsted, Minessota, que cuenta con 144.248 habitantes, obteniendo una incidencia de 4 casos nuevos por 100.000 habitantes/año y una prevalencia de 42 casos por 100.000 habitantes, concluyendo que es una enfermedad rara en esa región ${ }^{10}$. Si trasladáramos esa cifra a Chile, deberíamos tener más de 6.000 pacientes, lo que dista mucho de la realidad. Calculando con las cifras observadas en el área norte de Santiago, una prevalencia de 6,1/100.000 da alrededor de 1.000 pacientes portadores de $\mathrm{PC}$ en el país, que aunque parece más real, probablemente sobrepasa largamente el número de pacientes con diagnóstico establecido. La incidencia y prevalencia reportadas en otros países es mucho mayor que la obtenida por nuestros resultados ${ }^{11-21}$. Un grupo español publicó recientemente sus resultados, con una incidencia anual de 5,5/100.000 habitantes y una prevalencia 49,3/100.000 habitantes, es decir, 7 veces superior a nuestras cifras. Este grupo encontró $1 / 3$ de pacientes calcificados, al utilizar EUS para el diagnóstico, resultado similar al obtenido por nosotros en Hungría, utilizando pruebas directas de función pancreática ${ }^{11,15}$.

Es posible encontrar pacientes con $\mathrm{PC}$ entre los diabéticos. En efecto, hace 10 años estudiamos la función pancreática exocrina en un grupo de 70 diabéticos $^{7}$. Dos pacientes fueron previamente 
excluidos por pesquisar en ellos alteraciones morfológicas compatibles con PC avanzada. En el grupo estudiado encontramos $30 \%$ de pacientes con disminución de la elastasa fecal, que podrían corresponder a pacientes en fase inicial de PC. Estos pacientes continúan en control por diabetólogos y ninguno ha desarrollado PC.

Desde nuestro punto de vista es importante considerar las formas oligosintomáticas de la enfermedad ${ }^{22}$ que, asociadas a las deficiencias del sistema, llevan a una menor consulta y menos esfuerzos diagnósticos. La vieja polémica continúa: ¿Es la enfermedad silente o el médico sordo?

Sin embargo, aun cuando no caben dudas que hay falencias de diagnóstico, parece ser que la PC efectivamente es una enfermedad infrecuente en Chile.

Quizás la explicación se encuentra en la población sana. En efecto, la población chilena bebe menos alcohol y fuma en menor cantidad en comparación con países europeos, Estados Unidos de Norteamérica (USA) y Japón ${ }^{23,24}$ (Tabla 3). Sin embargo, las estadísticas de la Organización Mundial de la Salud (OMS) no muestran diferencias significativas con los datos de México y Brasil donde la prevalencia de PC es mucho mayor. Una diferencia podría encontrarse en la calidad del alcohol: aparte de similar consumo de vino y cerveza, en México y Brasil se consumen bebidas de mayor graduación alcohólica. Los hombres en Brasil, además, inician su consumo alcohólico a temprana edad ${ }^{1,2}$. A pesar de lo anterior, el concepto aceptado es que lo relevante es la cantidad de alcohol ingerido, más que el tipo de bebida ${ }^{25}$.

$\mathrm{Al}$ analizar las etiologías, es llamativa la falta de etiología identificable en mujeres, lo que podría relacionarse a una entrega parcial de información respecto del consumo alcohólico en este grupo. El tabaco es un factor de riesgo independiente de $\mathrm{PC}$, sin embargo, su consumo en nuestro grupo (2/3 de los pacientes) fue similar al de la población general.

La desnutrición infantil es importante en la patogenia de la pancreatitis tropical, que no ha sido descrita en nuestro país. En Chile, la desnutrición infantil no es un problema en las últimas décadas $^{26}$. Mientras el consumo de alcohol asociado a desnutrición proteica es un factor de riesgo de cirrosis hepática, cuando se asocia a dietas ricas en grasa y proteína, es un factor de riesgo de $\mathrm{PC}^{25}$. En Hungría encontramos un notorio aumento en
Tabla 3. Tabla 3. Consumo de tabaco y alcohol en diferentes países de América Latina y del mundo según los datos de $\mathrm{OMS}^{22,23}$

\begin{tabular}{|lcc|}
\hline & $\begin{array}{c}\text { Cigarrillo } \\
\text { n/persona/ } \\
\text { año }\end{array}$ & $\begin{array}{c}\text { Alcohol puro } \\
\text { Litro/persona/ } \\
\text { año }\end{array}$ \\
\hline Argentina & 1.042 & 9,4 \\
Brasil & 504 & 10,3 \\
\hline Chile & $\mathbf{8 6 0}$ & $\mathbf{8 , 8}$ \\
\hline Colombia & 412 & 6,6 \\
\hline Ecuador & 227 & 9,4 \\
\hline México & 371 & 8,6 \\
\hline Uruguay & 770 & 9,0 \\
\hline España & 1.757 & 11,8 \\
\hline Francia & 854 & 12,5 \\
\hline Países Bajos & 801 & 9,9 \\
\hline Alemania & 1.045 & 11,8 \\
\hline Hungría & 1.518 & 16,1 \\
\hline Polonia & 1.586 & 14,4 \\
\hline Republica Checa & 2.125 & 16,5 \\
\hline Inglaterra & 750 & 11,6 \\
\hline Estados Unidos & 1.028 & 9,2 \\
\hline Japón & 1.841 & 7,2 \\
\hline S núneros & & 16 \\
\hline
\end{tabular}

Los números muestran consumo de cigarrillos y el promedio de consumo de alcohol en población mayor de 15 años. No hay diferencia importante entre los datos de Chile y otros países vecinos, en particular, consumo en Brasil y México.

la incidencia y prevalencia de PC 20 años después de producirse una mejoría en el estándar de vida y en el consumo de proteínas ${ }^{15}$. Es posible que en Chile ocurra un fenómeno similar ${ }^{27}$, considerando que, a pesar de las bajas cifras, el diagnóstico ha ido en aumento.

Otro factor llamativo a considerar fue la baja prevalencia de pacientes con etnia Mapuche en nuestro grupo. Chile es una mezcla genética entre europeos y amerindios. Los Mapuches representan según algunas fuentes entre el 4\%-10\% de la población, encontrando genes mapuches casi en el $50 \%$ de la población chileno-hispana ${ }^{28}$. Es bien sabido que la colelitiasis es muy frecuente en mapuches. Miquel y cols. encontraron el sustrato metabólico de este fenómeno cuya base sería 
genética $^{29}$. La litiasis biliar y la enfermedad hepática alcohólica se asocian raramente con PC $^{30,31}$. Tomando en cuenta estos hechos, nos parece razonable plantear la posibilidad de que los genes mapuches confieran cierto grado de protección contra el desarrollo de PC.

Las limitaciones de nuestro estudio son su carácter retrospectivo y nuestra incapacidad para diagnosticar precozmente la enfermedad. Además, podemos estar subestimando las cifras al no poder considerar a los pacientes que se atienden en otros centros y los que no consultan.

En conclusión, con seguridad la PC es una enfermedad subdiagnosticada en Chile, en parte por su evolución silente en un grupo de pacientes y por nuestras limitaciones en el estudio de los pacientes que consultan. Nuestras experiencias hacen probable un aumento en la incidencia y prevalencia de la enfermedad, aun así, las cifras son bajas en nuestro país. Los factores dietéticos y el consumo de alcohol y tabaco no bastan para explicar estas diferencias con otras regiones del mundo, pudiendo haber factores genéticos implicados.

\section{Referencias}

1. Dani R, Mott CB, Guarita DR, Nogueira CE. Epidemiology and etiology of chronic pancreatitis in Brazil: a tale of two cities. Pancreas 1990; 5: 474-8.

2. Nogueira CE, Dani R. Some features of chronic pancreatitis in Brazil, especially in the city of Belo Horizonte. In: Pancreatitis. New data and geographical distribution. Sarles H, Johnson CD, Saunière JF (Eds). Arnette-Blackwell, Paris 1991; pp. 203-11.

3. Robles-Díaz G, García-Tsao G. Chronic pancreatitis in Mexico City: comparison with chronic liver disease. In: Pancreatitis. New data and geographical distribution. Sarles H, Johnson CD, Saunière JF (Eds). Arnette-Blackwell, Paris 1991; pp. 197-202.

4. Sarles H, Cros RC, Bidart JM. A multicenter inquiry into the etiology of pancreatic diseases. Digestion 1979; 19: 110-25.

5. Rossi R, de Aretxabala X, Watkins G, Navarrete C, Vargas L, Accatino L, et al. Pancreatitis crónica; Una experiencia nacional reciente de su manejo quirúrgico. Rev Med Chile 1997; 125: 911-6.

6. Alonso FT, Garmendia ML, De Aguirre M, Searle J. Análisis de la tendencia de la mortalidad por cirrosis hepática en Chile: Años 1990 a 2007. Rev Med Chile 2010; 138: 1253-8.
7. Mancilla C, Hurtado C, Tobar E, Orellana NI, Pineda BP, Castillo MI, et al. Función pancreática exocrina en diabetes mellitus: Determinación de elastasa fecal. Rev Med Chile 2006; 134: 407-14.

8. Guía Clínica Diabetes Mellitus tipo 2. Santiago, Minsal, 2010. ISBN: 978-956-8823-48-1, http://web.minsal.cl/ portal/url/item/72213ed52c3e23d1e04001011f011398. pdf, Fecha de publicación: enero de 2010 (consultado el 20 de agosto de 2016).

9. Conwell DL, Lee SL, Yadav D, Longnecker DS, Miller FH, Mortele KJ, et al. American Pancreatic Association Practice Guidelines in Chronic Pancreatitis. Evidence-Based Report on Diagnostic Guidelines. Pancreas 2014; 43: 1143-62.

10. Yadav D, Timmons L, Benson JT, Dierkhising RA, Chari ST. Incidence, prevalence, and survival of chronic pancreatitis: a population-based study. Am J Gastroenterol 2011; 106: 2192-9.

11. Domínguez-Muñoz JE, Lucendo A, Carballo F, Iglesias-García J, Tenías JM. A Spanish multicenter study to estimate the prevalence and incidence of chronic pancreatitis and its complications. Rev Esp Enferm Dig 2014; 106: 239-45.

12. Spanier BWM, Bruno MJ, Dijkgraaf MGW. Incidence and mortality of acute and chronic pancreatitis in the Netherlands: A nationwide record-linked cohort study for the years 1995-2005 World J Gastroenterol 2013; 19: 3018-26.

13. Andersen BN, Pedersen NT, Scheel J, Worning H. Incidence of alcoholic chronic pancreatitis in Copenhagen. Scand J Gastroentrol 1982; 17: 247-52.

14. Bang UC, Benfield T, Hyldstrup L, Bendtsen F, Beck Jensen JE. Mortality, Cancer, and Comorbidities Associated With Chronic Pancreatitis: A Danish Nationwide Matched-Cohort Study. Gastroenterology 2014; 146: 989-94.

15. Berger Z, Pap A. Chronic pancreatitis in Hungary. In: Pancreatitis. New data and geographical distribution. Sarles H, Johnson CD, Saunière JF (Eds). Arnette-Blackwell, París 1991; pp 185-90.

16. Gullo L. Chronic pancreatitis in Italy In: Pancreatitis. New data and geographical distribution. Sarles H, Johnson CD, Saunière JF (Eds). Arnette-Blackwell, Paris 1991; pp 157-65.

17. Lévy P, Barthet M, Mollard BR, Amouretti M, Marion-Audibert AM, Dyard F. Estimation of the prevalence and incidence of chronic pancreatitis and its complications. Gastroenterol Clin Biol 2006; 30: 83844.

18. Dite P, Stary K, Novotny I, Precechtelová M, Dolina $\mathrm{J}$, Lata J, et al. Incidence of chronic pancreatitis in the 
Czech Republic. European Journal of Gastroenterology \& Hepatology 2001; 13: 749-50.

19. Dufour MC, Adamson MD. The epidemiology of alcohol-induced pancreatitis. Pancreas 2003; 27: 286-90.

20. Jupp J, Fine D, Johnson CD. The epidemiology and socioeconomic impact of chronic pancreatitis. Best Pract Res Clin Gastroenterol 2010; 24: 219-31.

21. Hirota M, Shimosegawa T, Masamune A, Kikuta K, Kume K, Hamada S, et al. The seventh nationwide epidemiological survey for chronic pancreatitis in Japan: Clinical significance of smoking habit in Japanese patients. Pancreatology 2014; 14; 490-6.

22. Forsmark CE. Management of Chronic Pancreatitis. Gastroenterology 2013; 144: 1282-91.

23. Global status report on alcohol and health $2014 \mathrm{WHO}$ http://www.who.int/substance_abuse/publications/ global_alcohol_report/en/ (consultado el 12 de abril de 2016).

24. The Tobacco Atlas. http://www.tobaccoatlas.org/products/cigarette_consumption/annual_cigarette_consumption/ consultado el 22 de julio de 2014.

25. Durbec JP, Sarles H. Multicenter survey of the etiology of pancreatic diseases. Relationship between the relative risk of developing chronic pancreatitis and alcohol, protein and lipid consumption. Digestion 1978; 18 : 337-50.

26. Olivares S, Zakarias H. Informe final. Estudio para revisión y actualización de las guías alimentarias para la población Chilena http://www.dinta.cl/wp-dintacl/ wp-content/uploads/Estudio_revision_actualizaci\%C3\%B3n_Guias_Alimentarias_Chile2013.pdf (consultado el 12 de abril de 2016).

27. Encuesta Nacional de consumo alimentario. Informe final. http://web.minsal.cl/sites/default/files/ENCA-INFORME_FINAL.pdf (consultado el 12 de abril de 2016).

28. Fuentes M, Pulgar I Gallo C, Bortolini M-C, Canizales-Quinteros S, Bedoya G et al: Geografía génica de Chile. Distribución regional de los aportes genéticos americanos, europeos y africanos. Rev Med Chile 2014; 142: 281-9.

29. Miquel JF, Covarrubias C, Villarroel L, Mingrone G, Greco AV, Puglielli L, et al. Genetic Epidemiology of Cholesterol Cholelithiasis Among Chilean Hispanics, Amerindians, and Maoris. Gastroenterology 1998; 115: 937-46.

30. Nakamura Y, Kobayashi Y, Ishikawa A, Maruyama K, Higuchi S. Severe chronic pancreatitis and severe liver cirrhosis have different frequencies and are independent risk factors in male Japanese alcoholics J Gastroenterol 2004; 39: 879-87.

31. Aparisi L, Sabater L, Del-Olmo J, Sastre J, Serra MA, Campello R, et al. Does an association exist between chronic pancreatitis and liver cirrhosis in alcoholic subjects? World J Gastroenterol 2008 October 28; 14: 6171-9. 